\title{
Applying EFT to Higgs pair production in universal extra dimensions
}

\section{Lisa Edelhäuser, Alexander Knochel and Thomas Steeger}

Institut für Theoretische Teilchenphysik und Kosmologie, RWTH Aachen, Sommerfeldstr. 16, Aachen, 52074 Germany

E-mail: ledelhaeuser@physik.rwth-aachen.de, knochel@physik.rwth-aachen.de, thomas.steeger@rwth-aachen.de

ABSTRACT: We investigate single Higgs and Higgs pair production at the LHC in models of Universal Extra Dimensions. After calculating the relevant cross sections, we use the UED model as a testing ground for the Effective Field Theory approach to physics beyond the Standard Model. We show how the UED contributions to Higgs production can be matched to a dimension- 6 operator. We then discuss the range of validity of this approach, in particular for Higgs pair production, and determine the sensitivity to the number of KK modes in the loop.

Keywords: Higgs Physics, Beyond Standard Model, Field Theories in Higher Dimensions, Effective field theories

ARXIV EPRINT: 1503.05078 


\section{Contents}

1 Introduction 1

2 LO Higgs production in a UED model 2

2.1 Single Higgs production 3

2.2 Higgs pair production 3

3 Effective theory description $\quad 4$

$\begin{array}{llr}4 & \text { Conclusions } & 7\end{array}$

\section{Introduction}

After the discovery of a Higgs boson at the LHC [1, 2], the precise determination of its properties is of paramount importance. Many of the couplings to gauge bosons and fermions are already being measured in single Higgs production. However, in order to discriminate between the weakly interacting Standard Model Higgs and alternative EWSB scenarios, it is crucial to measure the Higgs self-interaction [3-6] which so far has only been determined indirectly from the Higgs mass in a model-dependent fashion. An important process allowing a direct measurement of these couplings at the LHC is Higgs pair production.

An important difference between on-shell single and double Higgs production via gluon fusion is the invariant mass flowing through the quark loop at LO, which is fixed at $p^{2}=m_{h}^{2}$ in the first case, ${ }^{1}$ but is only constrained by the collider energy in the other. Therefore, double Higgs production can yield additional sensitivity to new physics contributing to gluon fusion which can affect the self coupling measurement. In this work we consider a Standard Model extension in which the Higgs self coupling remains unchanged at tree level, while the loop in double Higgs production potentially resolves additional heavy degrees of freedom. Concretely, we use a minimal Universal Extra Dimensions scenario [9], where for the purpose of this work we are mainly interested in the heavy quark spectrum and its couplings to the lightest Higgs.

If one is interested in a model-independent description of heavy new physics in Higgs production, the effective field theory (EFT) framework using dimension-6 operators [10-14] proves to be a very efficient tool. However, since the EFT expansion becomes invalid for invariant masses near the cutoff scales [15], comparisons with concrete UV completions are important in order to understand the precise scale and nature of this breakdown. We therefore match the new physics contributions to gluon fusion in our Universal Extra Dimensions (UED) scenario to the corresponding effective operator(s) and compare the

\footnotetext{
${ }^{1}$ See however the possibility to resolve the momentum dependence of the effective $g g h$-Vertex in gluon fusion via the radiation of additional jets [7] and off-shell Higgs measurements [8].
} 
results to the full 1-loop calculation. Higgs pair production has been calculated previously in such models, and we find a discrepancy between both our full 1-loop and EFT results and the published result in [16].

\section{LO Higgs production in a UED model}

Gluon fusion via (top) quark loops constitutes the dominant single and double Higgs pair production mode in the SM, and this remains true in the UED extension we consider here. We take into account the top quark and its Kaluza-Klein (KK) excitations in the loop. The concrete scenario we use is a simple version of mUED in which we neglect the mass splittings from loop effects and related boundary terms as higher order effects. Starting point is a $5 \mathrm{D}$ version of the SM Lagrangian compactified on a circle of radius $R$ in which each chiral SM fermion is extended to a full Dirac spinor. A $\mathbb{Z}_{2}$ orbifold symmetry reduces the extra dimension to an interval of length $y \in[0, \pi R]$ with orbifold fixed points at the boundaries $y=0, \pi R$. Its main purpose is to project out lefthanded singlets, righthanded doublets and the $V_{5}$ components of gauge bosons from the massless spectrum in order to recover the chiral SM in the low energy limit. The doubling of the fermionic field content in the 5D theory yields two Dirac type partners at each massive KK level for each SM Dirac fermion. For the top we denote them by $t_{1}^{n}$ and $t_{2}^{n}$ respectively. They are degenerate in mass at tree level. Details about this construction can be found in $[9,17]$.

These two degenerate top partners per level $t_{1}^{n}$ and $t_{2}^{n}$ receive a mass

$$
m_{\mathrm{t}, \mathrm{KK}}=\sqrt{m_{\mathrm{top}}^{2}+n^{2} / R^{2}}
$$

which includes an SM-like contribution $m_{\text {top }}$ from the Higgs mechanism and a geometric contribution $n / R$ from the momentum in the extra dimension. Their couplings to the lightest Higgs and gluons are shown in figure 1. Note that due to gauge invariance, the coupling of these KK-top quarks to the massless gluon is identical to that of the SM top quark, while the couplings to the Higgs boson differ between the SM particles and their KK modes: in our basis choice, there is a non-mixing contribution which is suppressed by the KK mass, but yields an SM-like coupling for the zero mode

$$
\frac{m_{\mathrm{top}}^{2}}{v \sqrt{m_{\mathrm{top}}^{2}+n^{2} / R^{2}}} \stackrel{n=0}{\longrightarrow} \frac{m_{\mathrm{top}}}{v},
$$

as well as a mixing contribution whose coupling converges to the SM yukawa coupling for large mode numbers,

$$
\frac{m_{\mathrm{top}} \frac{n}{R}}{v \sqrt{m_{\mathrm{top}}^{2}+n^{2} / R^{2}}} \stackrel{n \rightarrow \infty}{\longrightarrow} \frac{m_{\mathrm{top}}}{v} .
$$

These relations will become important in the EFT matching later. Due to the mass degeneracy of the two KK top modes, these couplings can in principle be completely diagonalized if desired, but we choose to keep the basis with mixing and non-mixing Feynman Rules as given above to keep the hierarchy of suppression manifest. Let us now turn to the matrix elements for single and double Higgs production in this model. 

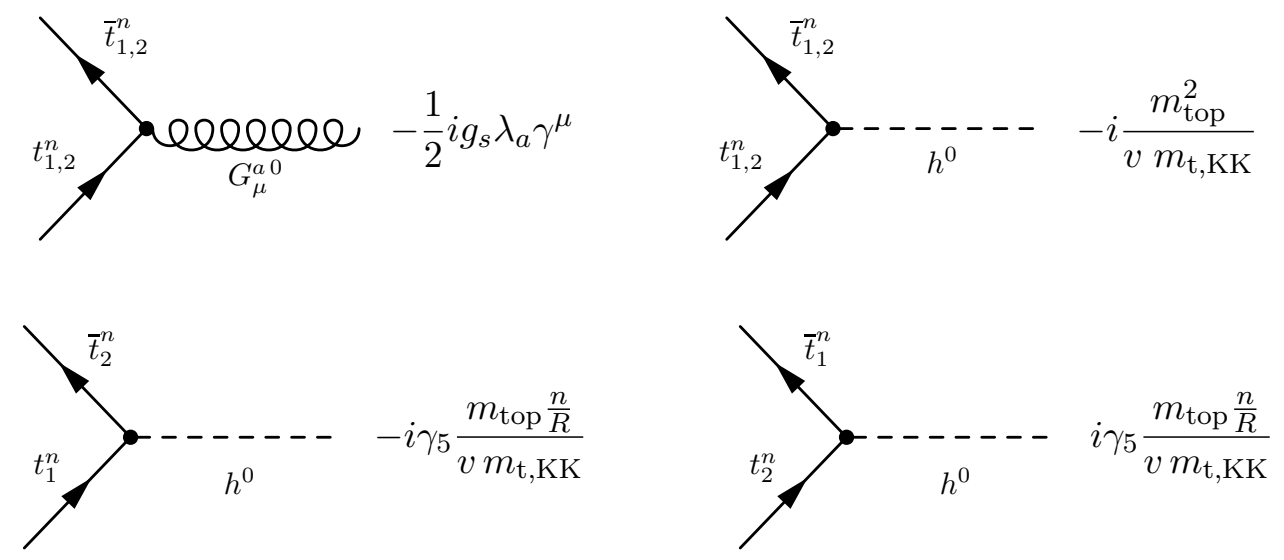

Figure 1. The relevant Feynman rules for Higgs production via gluon fusion in our UED scenario. Here, $\lambda_{a}$ denote the usual Gell-Mann matrices, $v$ and $g_{s}$ are the $4 \mathrm{D}$ values of the vacuum expectation value and the strong coupling respectively. Furthermore, $n \in \mathbb{N}_{0}$ denotes the KK mode number. For $n=0$, which corresponds to the SM particles which obtain all mass via the Higgs mechanism, only $t=t_{1}^{0}$ exists while $t_{2}^{0}$ is projected out by the orbifold.

\subsection{Single Higgs production}

The Feynman diagram (up to crossing) contributing to this process in UED is a straightforward extension of the SM case [18] with an added KK index,

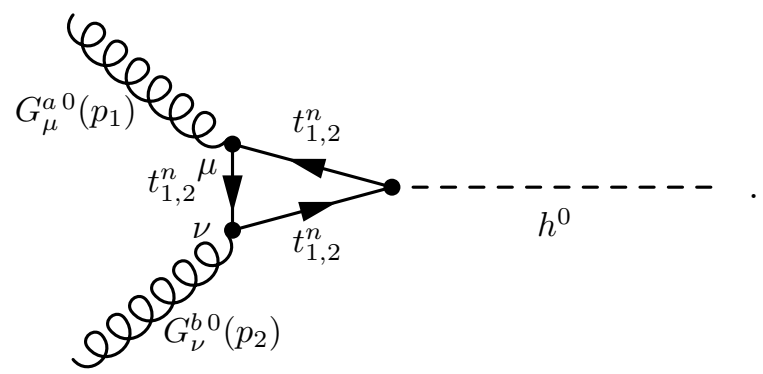

Note that the respective contributions of $t_{1}^{n}$ and $t_{2}^{n}$ to the loop are equal. We evaluate the matrix elements analytically with FeynCalc [19], the scalar integrals are then evaluated numerically with LoopTools [20]. Hadronic cross sections are obtained using the MSTW 2008 NLO PDFs [21]. Figure 2 shows the hadronic cross sections for a proton collider with $\sqrt{s}=13 \mathrm{TeV}$ at leading order (LO), contrasting single Higgs production in the SM with the UED scenario. We consider the first two KK-top quarks $(n=2)$ in the loop and show the dependence of the hadronic cross section on the inverse radius $R^{-1}$. The cross section is enhanced in the UED scenario and approaches the SM value from above for large values of $R^{-1}$ as the KK-top quarks become heavy and decouple from the theory. Single Higgs production in UED has previously been calculated in [22], and our results are compatible for $n \rightarrow \infty$.

\subsection{Higgs pair production}

In Higgs pair production one finds a couple of additional diagrams with mixing vertices compared to the SM case [23]. They are shown in figure 3. The first two diagrams $\left(A_{1}, A_{2}\right)$ 


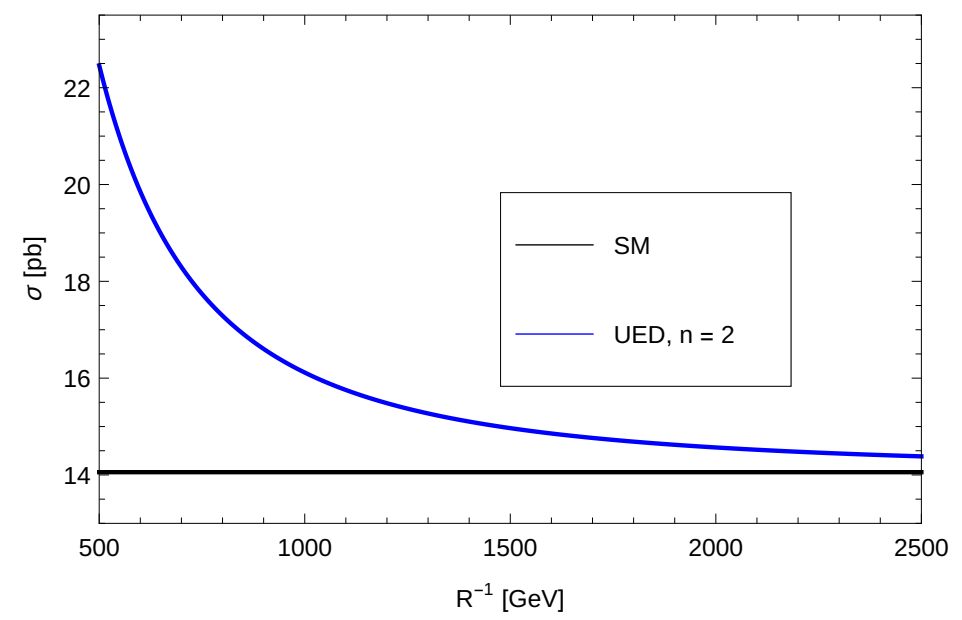

Figure 2. Hadronic LO cross sections for single Higgs production $p p \rightarrow h$ at $\sqrt{s}=13 \mathrm{TeV}$ in the SM and in UED including the first two KK-levels $(k \leq 2)$.

are topologically distinct non-mixing diagrams and thus straightforward generalizations of the SM case, while $A_{3}, A_{4}$ are a feature of the UED extension as they mix the two top partners of each massive KK-level. The last diagram $\left(A_{5}\right)$ describes production of a single offshell Higgs splitting into two final state Higgs particles, and again corresponds to the analogous SM contribution.

Figure 4 again compares the hadronic cross sections in the SM and in UED for a proton collider with $\sqrt{s}=13 \mathrm{TeV}$. Only the first two excitations $(n=2)$ have been taken into account for UED. The renormalization/factorization scale has been set to twice the Higgs mass $Q=2 m_{\mathrm{h}}$. In contrast to single Higgs production we observe an $R^{-1}$-dependent reduction of the cross section compared to the SM.

\section{Effective theory description}

Single on-shell Higgs production in the SM can be described to good precision by the effective Lagrangian obtained from integrating out the top quark,

$$
\sim G_{\mu \nu}^{a} G^{\mu \nu, a} v^{2} \ln (1+h / v)=G_{\mu \nu}^{a} G^{\mu \nu, a}\left(v h-\frac{h^{2}}{2}\right)+O\left(h^{3}\right) .
$$

This effective Lagrangian works well because $\sqrt{\hat{s}}=m_{h}<2 m_{t}$ for on-shell production. However, it quickly ceases to be useful for double Higgs production in which $\sqrt{\hat{s}} \geq 2 m_{h}$ very quickly exceeds $2 m_{t}$, leading to a breakdown of the heavy top approximation. New physics in double Higgs production on the other hand can be sufficiently heavy to allow for a perturbative effective theory description. Unlike the (linear) gauge symmetry breaking top quark contribution 3.1, the KK quark modes of our UED model consistently match to a gauge invariant dimension-6 operator

$$
\mathcal{O}_{G G}=G_{\mu \nu}^{a} G^{\mu \nu, a}\left(\phi^{\dagger} \phi-\frac{v^{2}}{2}\right)=G_{\mu \nu}^{a} G^{\mu \nu, a}\left(v h+\frac{h^{2}}{2}\right)
$$




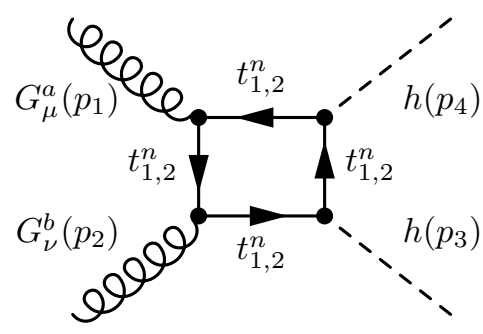

$A_{1}$

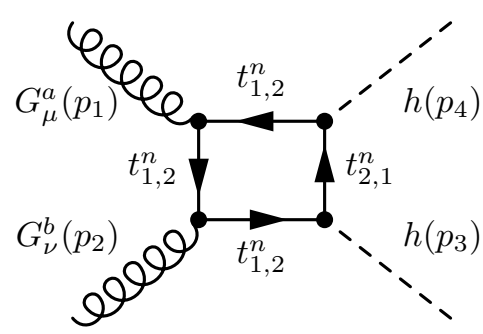

$A_{3}$

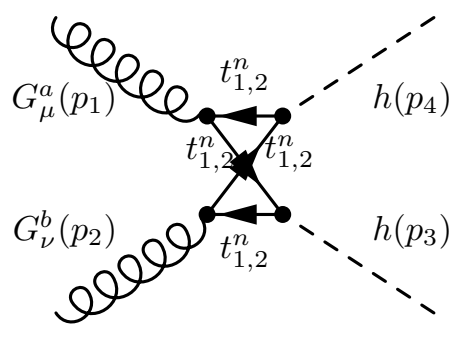

$A_{2}$

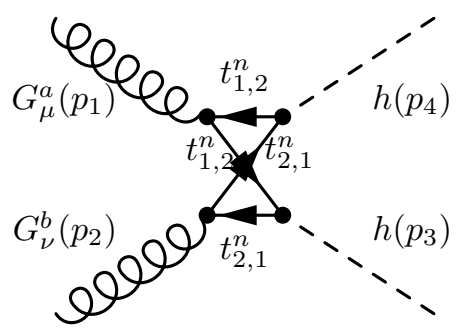

$A_{4}$

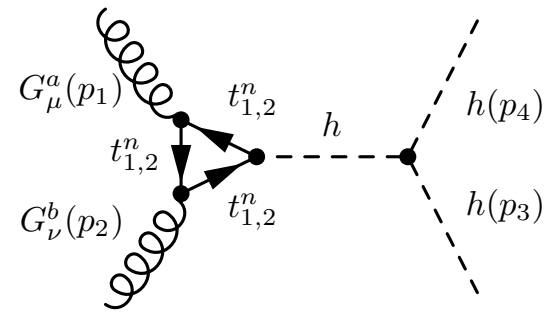

$A_{5}$

Figure 3. The diagrams (up to crossing) contributing to Higgs pair production.

(in unitary gauge) where we have subtracted the vacuum contributions to the gluon field strength normalization for convenience. Note that in the SM effective theory in eq. (3.1) we find a relative sign between the single and double Higgs couplings which is absent in the dimension-6 operator. We normalize the Wilson coefficient of the gauge invariant operator according to

$$
\mathcal{L}=\mathcal{L}_{\mathrm{SM}}+c \mathcal{O}_{G G} .
$$

The resulting Feynman rules are shown in figure 5. Higgs pair production in effective theories has previously been studied in $[24-26] .{ }^{2}$ Our aim is now to compare this approach to a concrete new physics scenario. This issue has been addressed recently for extended Higgs sectors in [27]. Since both the contributions to single and double Higgs production originate from the same gauge invariant operator, we only need to determine the matching constant $c$ once for one of the 1PI graphs. We hence carry out the matching procedure

\footnotetext{
${ }^{2}$ There seems to be a relative sign convention in the Wilson coefficient of $\mathcal{O}_{G G}$ between [24] and [25]. We find a reduced Higgs pair cross section for small positive Wilson coefficients, in accordance with [25].
} 


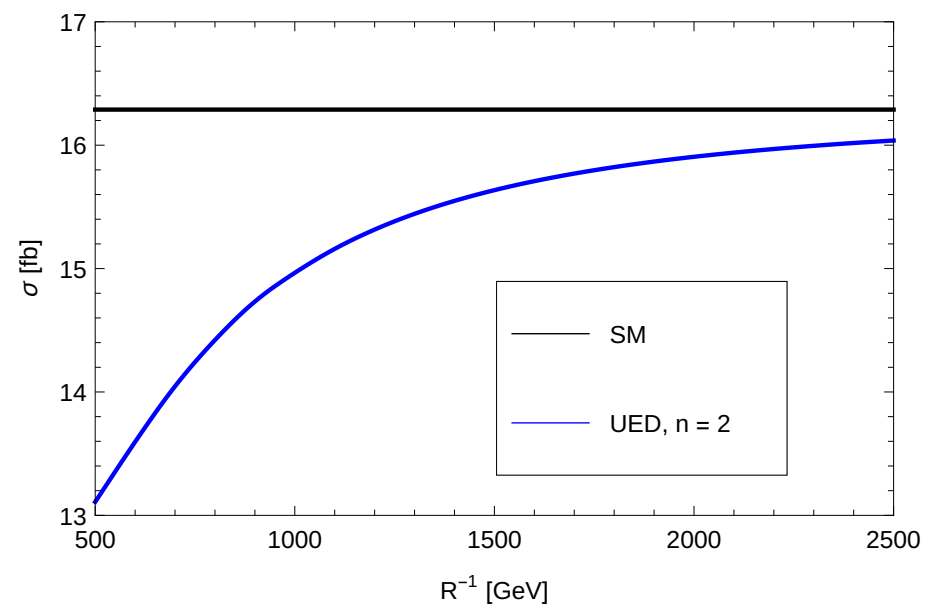

Figure 4. Hadronic cross sections for Higgs pair production $p p \rightarrow h h$ at $\sqrt{s}=13 \mathrm{TeV}$ in the SM and in UED including the first two KK-levels $(k \leq 2)$.

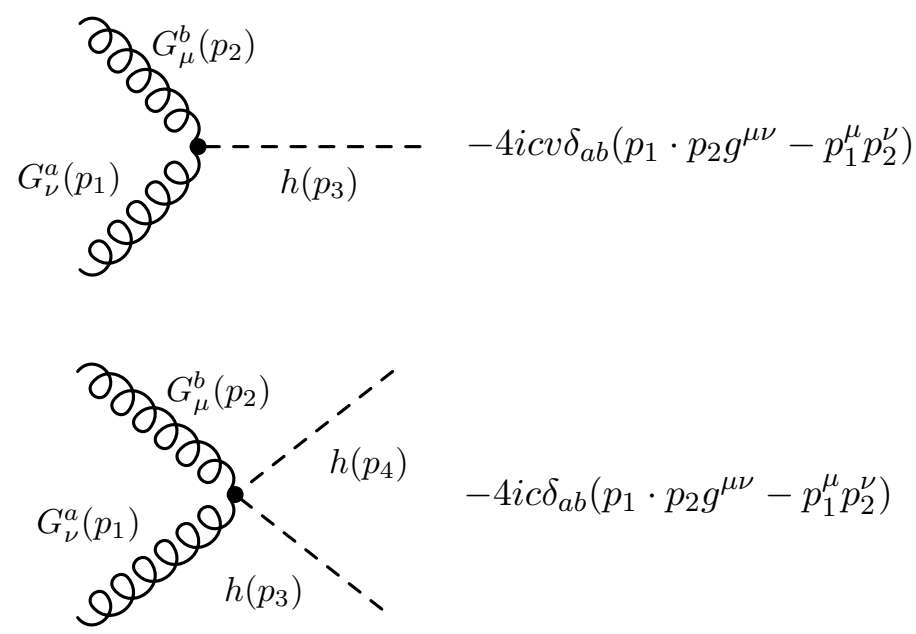

Figure 5. The additional Feynman rules for the $g g h$ and $g g h h$ vertices obtained from the operator $\mathcal{O}_{G G}$ in (3.3).

for the simpler case of the ggh coupling and then use the result to consistency check our double Higgs production calculation.

Unlike the top quark in the SM effective theory, the KK modes decouple for $m_{\mathrm{t}, \mathrm{KK}} \rightarrow \infty$ since their Yukawa couplings do not scale like their masses. It is therefore useful to think of the matching in terms of an expansion in $m_{\mathrm{t}, \mathrm{KK}}^{-1}$ to the leading nonvanishing order corresponding to an EFT expansion in $\Lambda^{-1} \sim m_{\mathrm{t}, \mathrm{KK}}^{-1}$, rather than a limit $m_{\mathrm{t}, \mathrm{KK}} \rightarrow \infty$. The matching constant naturally depends on the number of KK-excitations included in the loop. We obtain

$$
\begin{aligned}
c(n) & =\frac{g_{s}^{2} m_{\mathrm{top}}^{2}}{24 \pi^{2} v^{2}} \sum_{k=1}^{n} \frac{1}{m_{\mathrm{t}, \mathrm{KK}}^{2}}, \\
c(\infty) & =\frac{g_{s}^{2}}{48 \pi^{2} v^{2}}\left(\pi \frac{m_{\mathrm{top}}}{R^{-1}} \operatorname{coth}\left(\frac{\pi m_{\mathrm{top}}}{R^{-1}}\right)-1\right) .
\end{aligned}
$$


While the single and double Higgs interactions obtained from $\mathcal{O}_{G G}$ only differ by a factor of the vev $v$, the loop integrals in the 1PI-diagrams contributing to the two processes (namely fermion triangles and boxes respectively) have respective leading suppression scales $m_{\mathrm{t}, \mathrm{KK}}^{-1}$ and $m_{\mathrm{t}, \mathrm{KK}}^{-2}$. At first glance, this looks like it might spoil a consistent matching to a single gauge invariant dimension-6 operator. This discrepancy is however resolved by the scaling of the KK mode Yukawa couplings involved: in triangle diagrams, only nonmixing couplings can appear which themselves are suppressed with $m_{\mathrm{t}, \mathrm{KK}}^{-1}$, while the box diagrams $A_{3}, A_{4}$ contain unsuppressed mixing couplings. Hence, the leading contributions from triangles and boxes both scale as $m_{\mathrm{t}, \mathrm{KK}}^{-2}$. This also means that the non-mixing box diagrams only contribute to dimension- 8 operators and do not play a role in the leading order of EFT.

We use these results to again calculate single and double Higgs production cross sections, where the SM top quark contributions are now included as loop diagrams while all contributions from KK modes are absorbed into the effective vertex.

Figure 6 shows the relative deviation $\left(\sigma_{\mathrm{UED}}-\sigma_{\mathrm{SM}}\right) / \sigma_{\mathrm{SM}}$ between the SM and UED partonic double Higgs production cross sections. The UED KK mode contributions are included as 1-loop (solid) or EFT amplitudes (dashed). The simplicity of the EFT approximation allows to take the limit $n \rightarrow \infty$, which also yields a finite Wilson coefficient (making this particular process cutoff-independent at LO). In order to gauge the validity of the EFT approximation, we show a direct comparison of the 1-loop and effective contributions $\sigma_{e x}$ and $\sigma_{\text {eff }}$ in figure 7 . We notice that the EFT amplitudes increasingly overestimate the cross section as $\sqrt{\hat{s}}$ approaches $2 m_{\mathrm{t}, \mathrm{KK}}^{(1)} \approx 2 R^{-1}$, and underestimate them for $\sqrt{\hat{s}} \lesssim 500 \mathrm{GeV}$. This over- and underestimation hence tend to partially cancel in the total hadronic cross section, but will in principle be visible in differential distributions.

Figure 8 shows the hadronic single and pair production cross sections for $\sqrt{s}=13 \mathrm{TeV}$ as a function of $R^{-1}$. Here we compare the cross sections from the effective description to the SM cross section. We observe good agreement between our effective theory and the UED cross sections shown in figures 2 and 4.

\section{Conclusions}

We have revisited single and double Higgs production in a model of minimal Universal Extra Dimensions. While our results for single Higgs production are in accordance with those found in the literature, we find a discrepancy between existing results and our Higgs pair production cross sections. The aim of the second part was to match the loop contributions from Kaluza-Klein excitations of the top quark to the relevant effective dimension- 6 operator while keeping the SM top quark in the loop. We have performed this matching based on the hgg interaction and have then compared the full 1-loop results for Higgs pair production to the corresponding EFT cross sections. We find good agreement for low energies, which we interpret as a confirmation of our 1-loop results. For center-of-mass energies beyond the compactification scale, we find the expected increasing discrepancy between the full 1-loop and EFT Higgs pair production cross sections. For compactification scales

$R^{-1} \gtrsim 1 \mathrm{TeV}$, the EFT increasingly overestimates the cross sections for large $\sqrt{\hat{s}}$, but the 

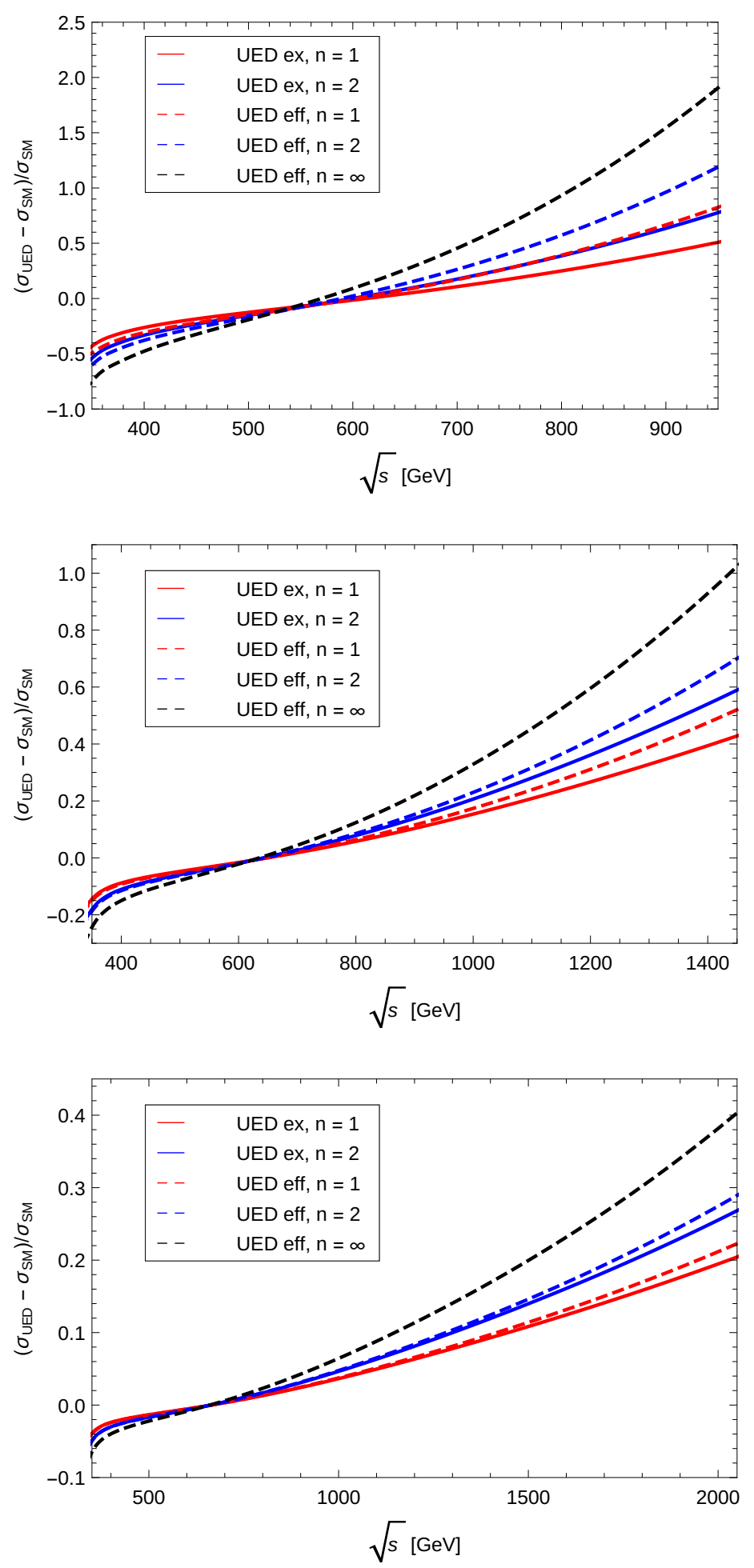

Figure 6. LO partonic double Higgs production cross sections normalized to the SM. The effects of the KK resonances are taken into account as 1-loop amplitudes (UED ex, solid) and as effective vertices (UED eff, dashed) respectively for $R^{-1}=500,1000,2000 \mathrm{GeV}$ from top to bottom. The result for $n \rightarrow \infty$ KK-modes is shown in the effective theory case only. 

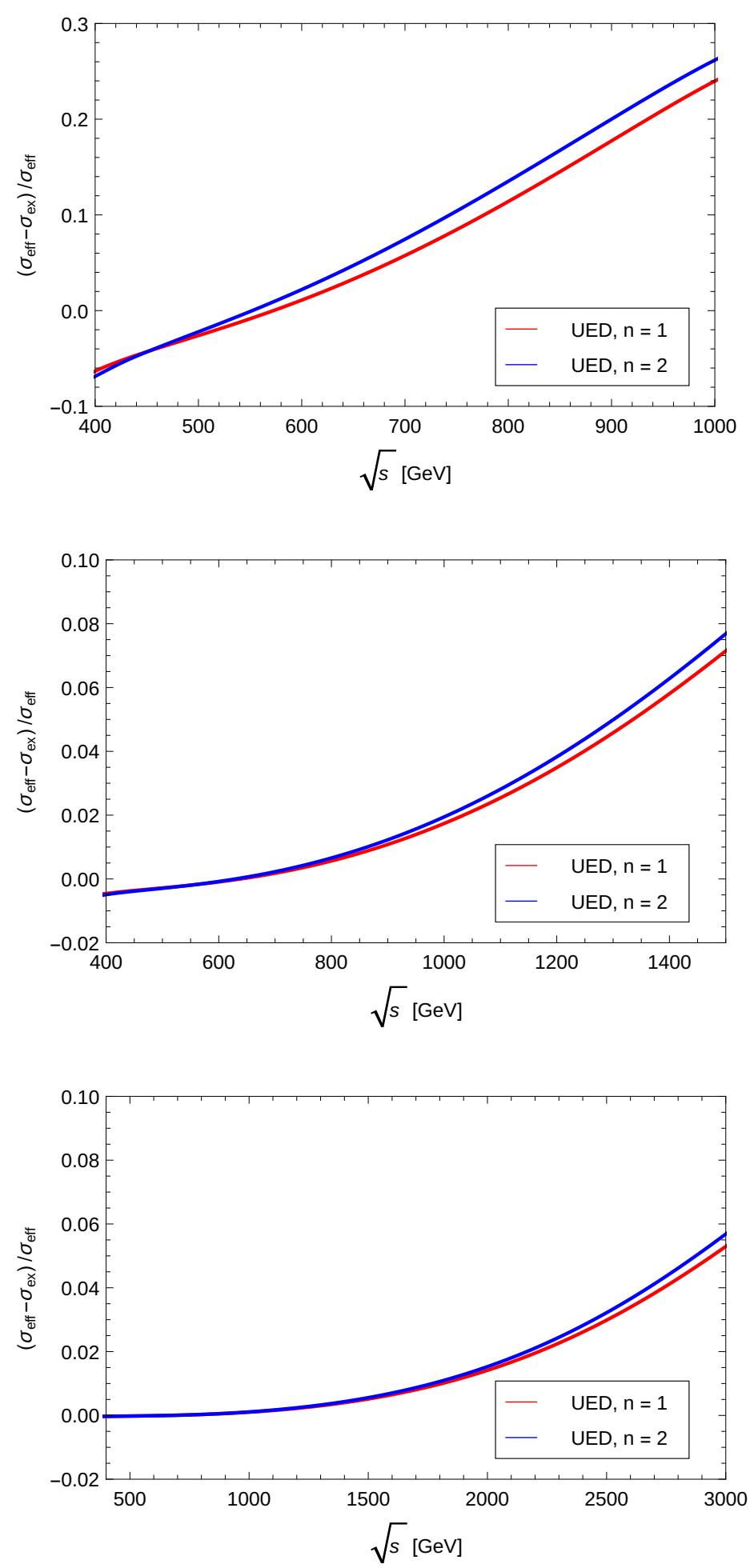

Figure 7. The difference of LO partonic and EFT double Higgs production cross sections normalized to the EFT with $R^{-1}=500,1000,2000 \mathrm{GeV}$ from top to bottom. 

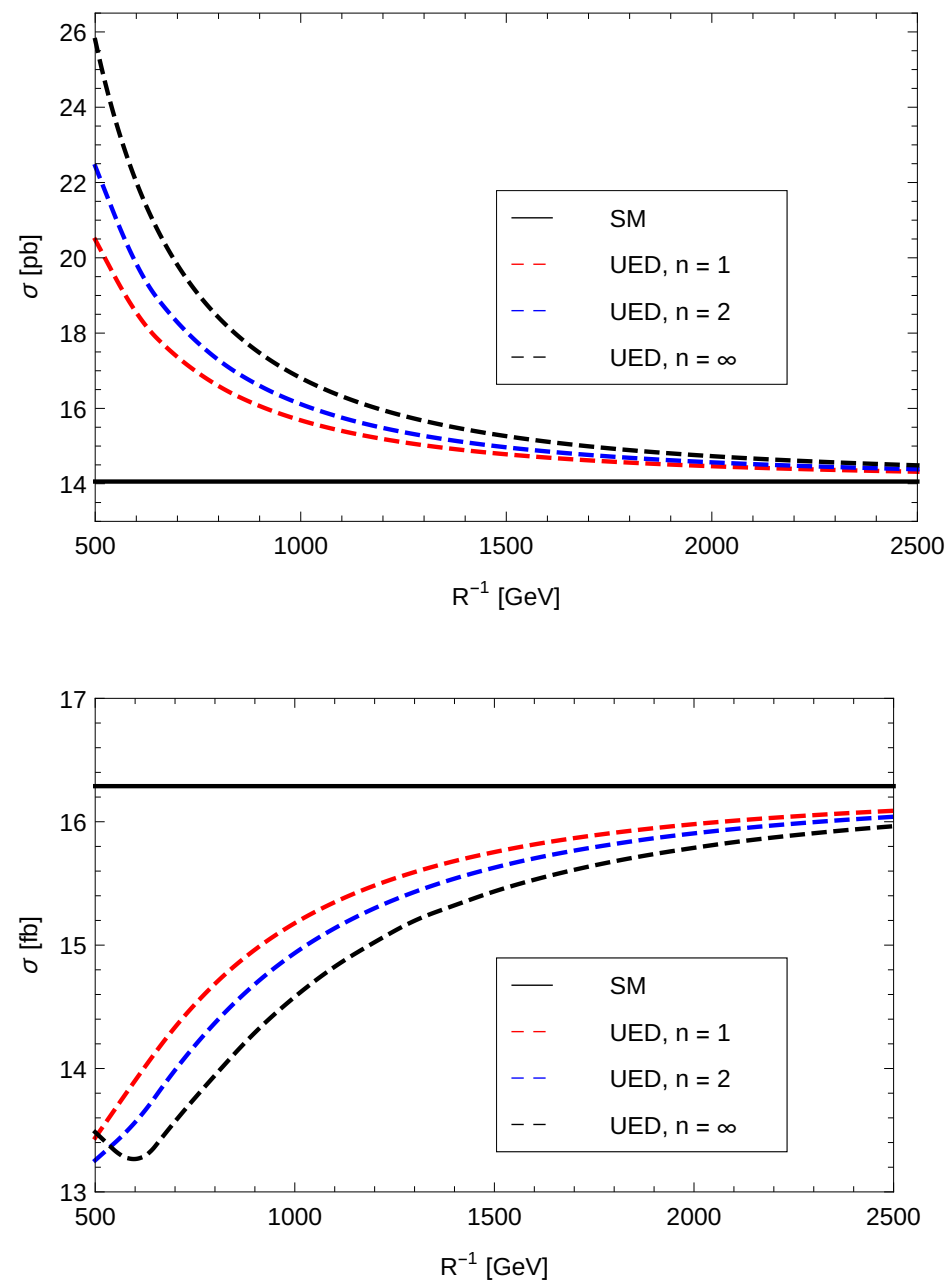

Figure 8. The hadronic cross sections for single and pair production at $\sqrt{s}=13 \mathrm{TeV}$ in the effective description including $n=1,2, \infty$ KK-excitations.

deviation remains below $2 \%$ for $\sqrt{\hat{s}} \lesssim R^{-1}$. For small $\sqrt{\hat{s}}$, the EFT underestimates the cross sections. For small compactification scales $R^{-1}=500 \mathrm{GeV}$, deviations are more pronounced. Nevertheless, the EFT approximation works well for $\mathrm{TeV}$ scale compactifications, and in particular for Higgs pair production at the LHC.

\section{Acknowledgments}

We thank M. Krämer and F. Riva for valuable discussions.

Open Access. This article is distributed under the terms of the Creative Commons Attribution License (CC-BY 4.0), which permits any use, distribution and reproduction in any medium, provided the original author(s) and source are credited. 


\section{References}

[1] CMS collaboration, Observation of a new boson at a mass of $125 \mathrm{GeV}$ with the CMS experiment at the LHC, Phys. Lett. B 716 (2012) 30 [arXiv:1207.7235] [InSPIRE].

[2] ATLAS collaboration, Observation of a new particle in the search for the Standard Model Higgs boson with the ATLAS detector at the LHC, Phys. Lett. B 716 (2012) 1 [arXiv:1207.7214] [INSPIRE].

[3] U. Baur, T. Plehn and D.L. Rainwater, Probing the Higgs selfcoupling at hadron colliders using rare decays, Phys. Rev. D 69 (2004) 053004 [hep-ph/0310056] [InSPIRE].

[4] U. Baur, T. Plehn and D.L. Rainwater, Examining the Higgs boson potential at lepton and hadron colliders: A Comparative analysis, Phys. Rev. D 68 (2003) 033001 [hep-ph/0304015] [INSPIRE].

[5] M.J. Dolan, C. Englert and M. Spannowsky, New Physics in LHC Higgs boson pair production, Phys. Rev. D 87 (2013) 055002 [arXiv: 1210.8166] [INSPIRE].

[6] M.J. Dolan, C. Englert and M. Spannowsky, Higgs self-coupling measurements at the LHC, JHEP 10 (2012) 112 [arXiv:1206.5001] [INSPIRE].

[7] M. Buschmann, C. Englert, D. Goncalves, T. Plehn and M. Spannowsky, Resolving the Higgs-Gluon Coupling with Jets, Phys. Rev. D 90 (2014) 013010 [arXiv:1405.7651] [INSPIRE].

[8] A. Azatov, C. Grojean, A. Paul and E. Salvioni, Taming the off-shell Higgs boson, J. Exp. Theor. Phys. 120 (2015) 354 [Zh. Eksp. Teor. Fiz. 147 (2015) 410] [arXiv:1406.6338] [INSPIRE].

[9] T. Appelquist, H.-C. Cheng and B.A. Dobrescu, Bounds on universal extra dimensions, Phys. Rev. D 64 (2001) 035002 [hep-ph/0012100] [InSPIRE].

[10] W. Buchmüller and D. Wyler, Effective Lagrangian Analysis of New Interactions and Flavor Conservation, Nucl. Phys. B 268 (1986) 621 [INSPIRE].

[11] J. Elias-Miro, J.R. Espinosa, E. Masso and A. Pomarol, Higgs windows to new physics through $D=6$ operators: constraints and one-loop anomalous dimensions, JHEP 11 (2013) 066 [arXiv: 1308.1879] [INSPIRE].

[12] T. Corbett, O.J.P. É boli, J. Gonzalez-Fraile and M.C. Gonzalez-Garcia, Determining Triple Gauge Boson Couplings from Higgs Data, Phys. Rev. Lett. 111 (2013) 011801 [arXiv: 1304.1151] [INSPIRE].

[13] A. Pomarol and F. Riva, Towards the Ultimate SM Fit to Close in on Higgs Physics, JHEP 01 (2014) 151 [arXiv: 1308.2803] [INSPIRE].

[14] B. Dumont, S. Fichet and G. von Gersdorff, A Bayesian view of the Higgs sector with higher dimensional operators, JHEP 07 (2013) 065 [arXiv: 1304.3369] [INSPIRE].

[15] A. Biekötter, A. Knochel, M. Krämer, D. Liu and F. Riva, Vices and virtues of Higgs effective field theories at large energy, Phys. Rev. D 91 (2015) 055029 [arXiv:1406.7320] [InSPIRE].

[16] H. de Sandes and R. Rosenfeld, Higgs pair production in models with universal extra dimensions, Phys. Lett. B 659 (2008) 323 [arXiv:0706.2665] [INSPIRE].

[17] J. Papavassiliou and A. Santamaria, Chiral fermions and gauge fixing in five-dimensional theories, Phys. Rev. D 63 (2001) 125014 [hep-ph/0102019] [INSPIRE]. 
[18] H.M. Georgi, S.L. Glashow, M.E. Machacek and D.V. Nanopoulos, Higgs Bosons from Two Gluon Annihilation in Proton Proton Collisions, Phys. Rev. Lett. 40 (1978) 692 [InSPIRE].

[19] R. Mertig, M. Böhm and A. Denner, FEYN CALC: Computer algebraic calculation of Feynman amplitudes, Comput. Phys. Commun. 64 (1991) 345 [INSPIRE].

[20] T. Hahn and M. Pérez-Victoria, Automatized one loop calculations in four-dimensions and D-dimensions, Comput. Phys. Commun. 118 (1999) 153 [hep-ph/9807565] [INSPIRE].

[21] A.D. Martin, W.J. Stirling, R.S. Thorne and G. Watt, Parton distributions for the LHC, Eur. Phys. J. C 63 (2009) 189 [arXiv:0901.0002] [InSPIRE].

[22] F.J. Petriello, Kaluza-Klein effects on Higgs physics in universal extra dimensions, JHEP 05 (2002) 003 [hep-ph/0204067] [INSPIRE].

[23] E.W.N. Glover and J.J. van der Bij, Higgs Boson Pair Production Via Gluon Fusion, Nucl. Phys. B 309 (1988) 282 [INSPIRE].

[24] A. Pierce, J. Thaler and L.-T. Wang, Disentangling Dimension Six Operators through Di-Higgs Boson Production, JHEP 05 (2007) 070 [hep-ph/0609049] [INSPIRE].

[25] F. Goertz, A. Papaefstathiou, L.L. Yang and J. Zurita, Higgs boson pair production in the $D=6$ extension of the SM, JHEP 04 (2015) 167 [arXiv:1410.3471] [INSPIRE].

[26] A. Azatov, R. Contino, G. Panico and M. Son, Effective field theory analysis of double Higgs boson production via gluon fusion, Phys. Rev. D 92 (2015) 035001 [arXiv: 1502.00539] [INSPIRE].

[27] M. Gorbahn, J.M. No and V. Sanz, Benchmarks for Higgs Effective Theory: Extended Higgs Sectors, arXiv:1502.07352 [INSPIRE]. 